\title{
Protée
}

\section{Alex Magrini : L’art qui désarme. Aux armes, et caetera}

\section{Ghislain Bourque}

Volume 29, numéro 1, 2001

La société des objets. Problèmes d'interobjectivité

URI : https://id.erudit.org/iderudit/030615ar

DOI : https://doi.org/10.7202/030615ar

Aller au sommaire du numéro

Éditeur(s)

Département des arts et lettres - Université du Québec à Chicoutimi

ISSN

0300-3523 (imprimé)

1708-2307 (numérique)

Découvrir la revue

Citer cet article

Bourque, G. (2001). Alex Magrini : L’art qui désarme. Aux armes, et caetera. Protée, 29(1), 48-54. https://doi.org/10.7202/030615ar d'utilisation que vous pouvez consulter en ligne.

https://apropos.erudit.org/fr/usagers/politique-dutilisation/ 
Ci-dessous: Alex Magrini, Et bien d'autres métiers, 2000. Médiums mixtes, $160 \times 120 \times 15 \mathrm{~cm}$. Page de droite: Alex Magrini, Triptyque vertical III, 2000-2001. M édiums mixtes, $80 \times 40 \times 15 \mathrm{~cm}$.
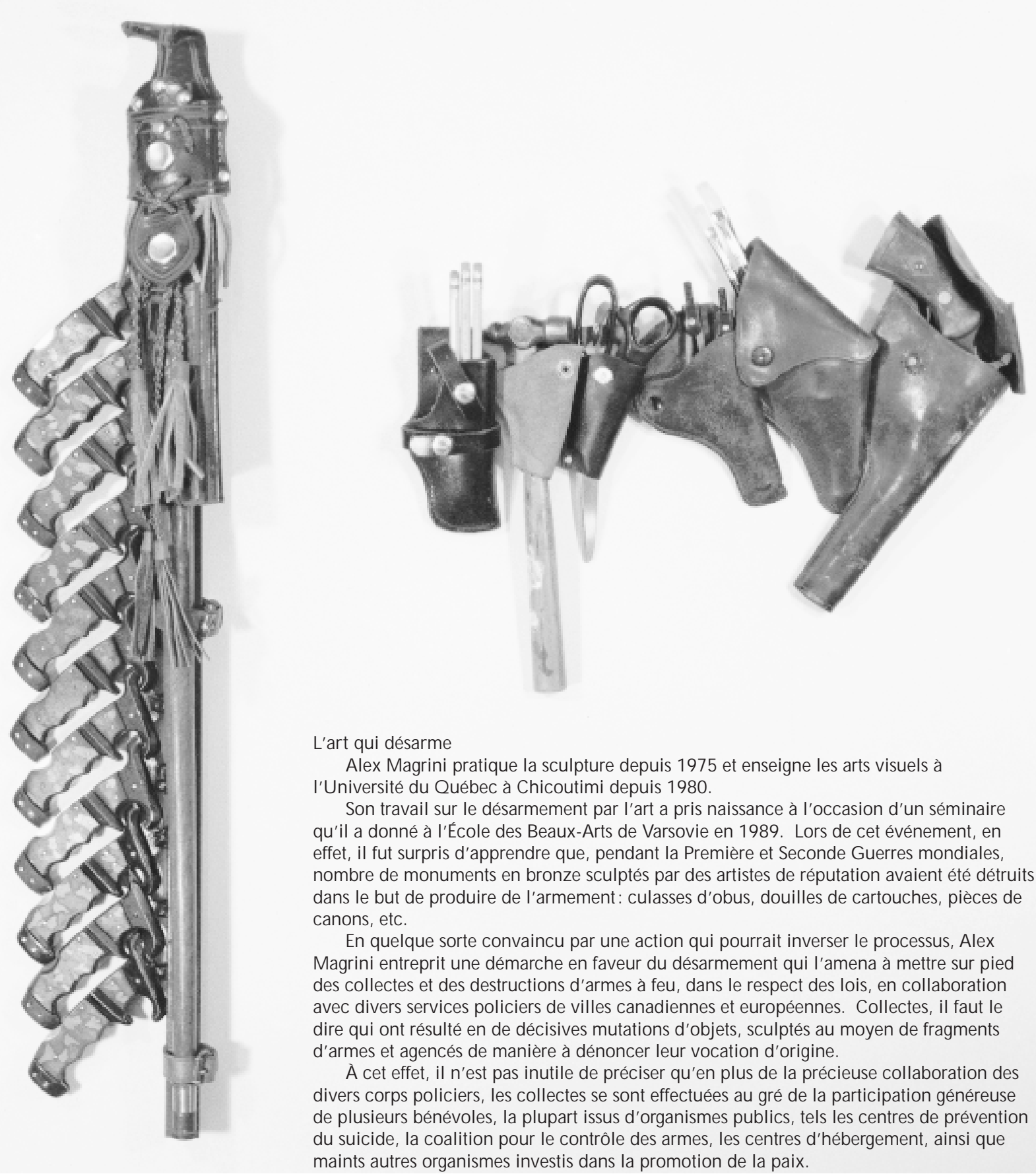

\section{L'art qui désarme}

Alex Magrini pratique la sculpture depuis 1975 et enseigne les arts visuels à

I'U niversité du Q uébec à Chicoutimi depuis 1980.

Son travail sur le désarmement par l'art a pris naissance à l'occasion d'un séminaire qu'il a donné à l'École des Beaux-Arts de Varsovie en 1989. Lors de cet événement, en effet, il fut surpris d'apprendre que, pendant la Première et Seconde Guerres mondiales, nombre de monuments en bronze sculptés par des artistes de réputation avaient été détruits dans le but de produire de l'armement: culasses d'obus, douilles de cartouches, pièces de canons, etc.

En quelque sorte convaincu par une action qui pourrait inverser le processus, Alex Magrini entreprit une démarche en faveur du désarmement qui l'amena à mettre sur pied des collectes et des destructions d'armes à feu, dans le respect des lois, en collaboration avec divers services policiers de villes canadiennes et européennes. Collectes, il faut le dire qui ont résulté en de décisives mutations d'objets, sculptés au moyen de fragments d'armes et agencés de manière à dénoncer leur vocation d'origine.

À cet effet, il n'est pas inutile de préciser qu'en plus de la précieuse collaboration des divers corps policiers, les collectes se sont effectuées au gré de la participation généreuse de plusieurs bénévoles, la plupart issus d'organismes publics, tels les centres de prévention du suicide, la coalition pour le contrôle des armes, les centres d'hébergement, ainsi que maints autres organismes investis dans la promotion de la paix.

Autre aspect important associé à cette cause, plusieurs artistes québécois, en collaboration (entre autres) avec l'Atelier d'Estampe Sagamie de Chicoutimi, ont mis à la disposition des agents collecteurs des centaines de gravures originales, faites pour être remises, en compensation, aux donateurs. 


\section{Aux armes, et cætera ...}

Criant de plaisir ses oublis, l'effeuilleuse s'écroula sous le tir en rafale de balles à blanc. Par un jeu d'éclairage contrôlé à distance, membre à membre, son corps finit par concéder à l'ombre sa noirceur. Avec pour résultat que, dans la vaste salle, un silence s'installa, à peine nourri des sons amortis de sa chute.

Dès lors, l'air de quelqu'une qui se serait trouvée éperdue, la sinueuse rombière reprit progressivement connaissance, ainsi que les pièces éparpillées de son luxuriant habit végétal. Pour se désennuyer un peu, les soirs de haute semaine, elle acceptait quelques balles contre une enfilade de mouvements assurés par sa chorégraphie écologique. Mais de ce cachet elle ne gardait rien, versant le tout au profit d'une cause humanitaire liée à I'ordre, la paix et la discipline.

À ce stade de sa prestation, le silence se faisait d'autant plus convaincant qu'elle avait su, juste avant sa chute finale, monter en crescendo une cascade de petits gloussements lancés à mesure que se détachaient ses malicieuses feuilles. Ce qui lui laissa le temps de disparaître en douce, derrière un rideau improvisé, où elle put rajuster son habit végétal, à l'abri des regards indiscrets.

Lorsqu'elle réapparut, la salle avait repris de la vigueur. Avec des proportions de multitude, les invités s'activaient à débattre sur les mots qui, comme les objets, se gardent toujours des munitions. Sitôt fatigués d'un sens, sur le champ, ils en font apparaître un autre, moins propre cela va de soi, mais y conférant les mesures d'hygiène appropriées.

Décalquée d'une lumière que I'on tenait parcimonieuse, elle devait désormais se consacrer à l'autre versant du travail pour lequel elle avait été embauchée. Ainsi, de l'une à l'autre table, se mêlant aux invités qui, à l'exclusion de toute autre femme, présentaient tous des faces farineuses, elle prenait appui sur le geste sacré du semeur pour lancer à la volée une flopée de petites gaufres cylindriques confectionnées dans un pain azyme pressé au plus sec. Et ils étaient nombreux à se bousculer pour seulement en saisir une avant qu'elle n'atteigne le sol, puisqu'en cas de réussite, ils gagnaient le privilège, de la main même de la vendeuse, sur le ventre et sur le dos, de se voir assener deux précis coups d'une frêle discipline de cuir dont les cordelettes étaient couronnées aux embouts de fines douilles en laiton vides de toute charge de plomb.

Dans une anarchie proche la haute voltige, la rombière n'accusait aucune fatigue, priant chacun de saisir ses oublies, tous suspendus aux virevoltes provoquées par ses tirs en rafale, chacun croulant sous la contrainte ouverte de ce singulier bal à blancs.

Ghislain Bourque,

Université du Q uébec à Chicoutimi 


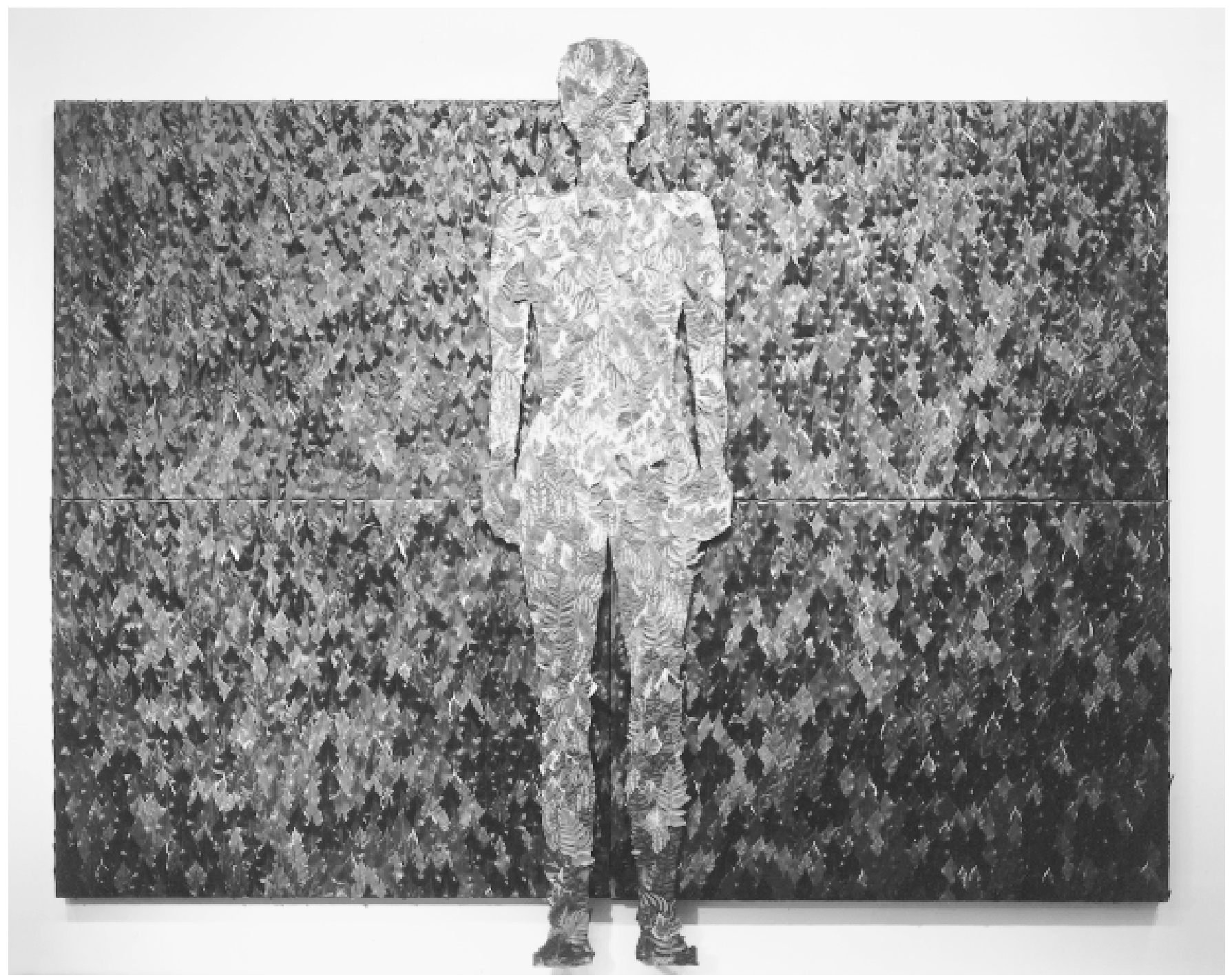

Alex Magrini, Camouflage, 1999. Bois et feuilles synthétiques, 170 x 190 × $30 \mathrm{~cm}$. 


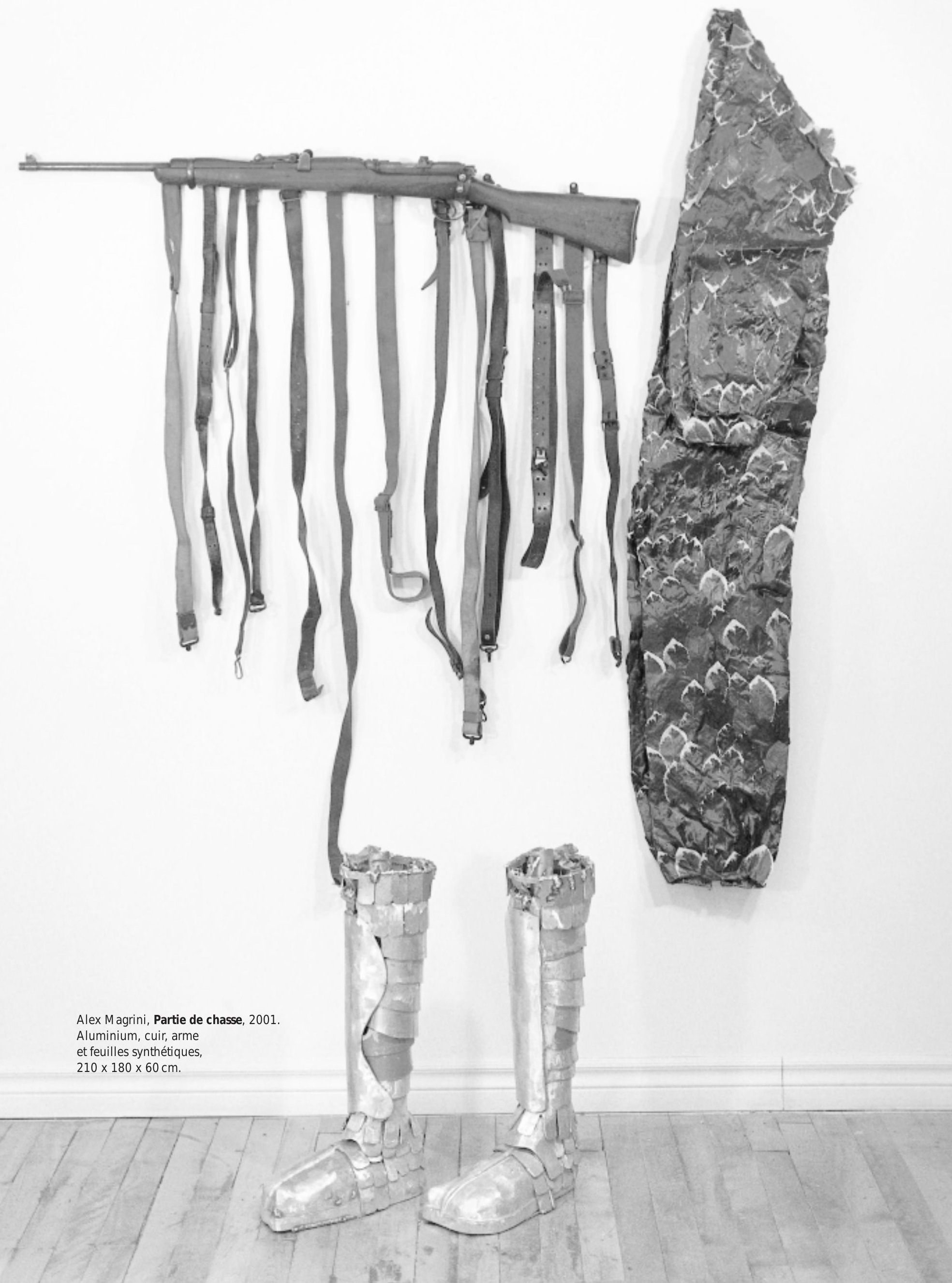






Alex Magrini, Boîtes de Pandore, 1998. Fragments d'armes et acier, $160 \times 220$ x $50 \mathrm{~cm}$. 


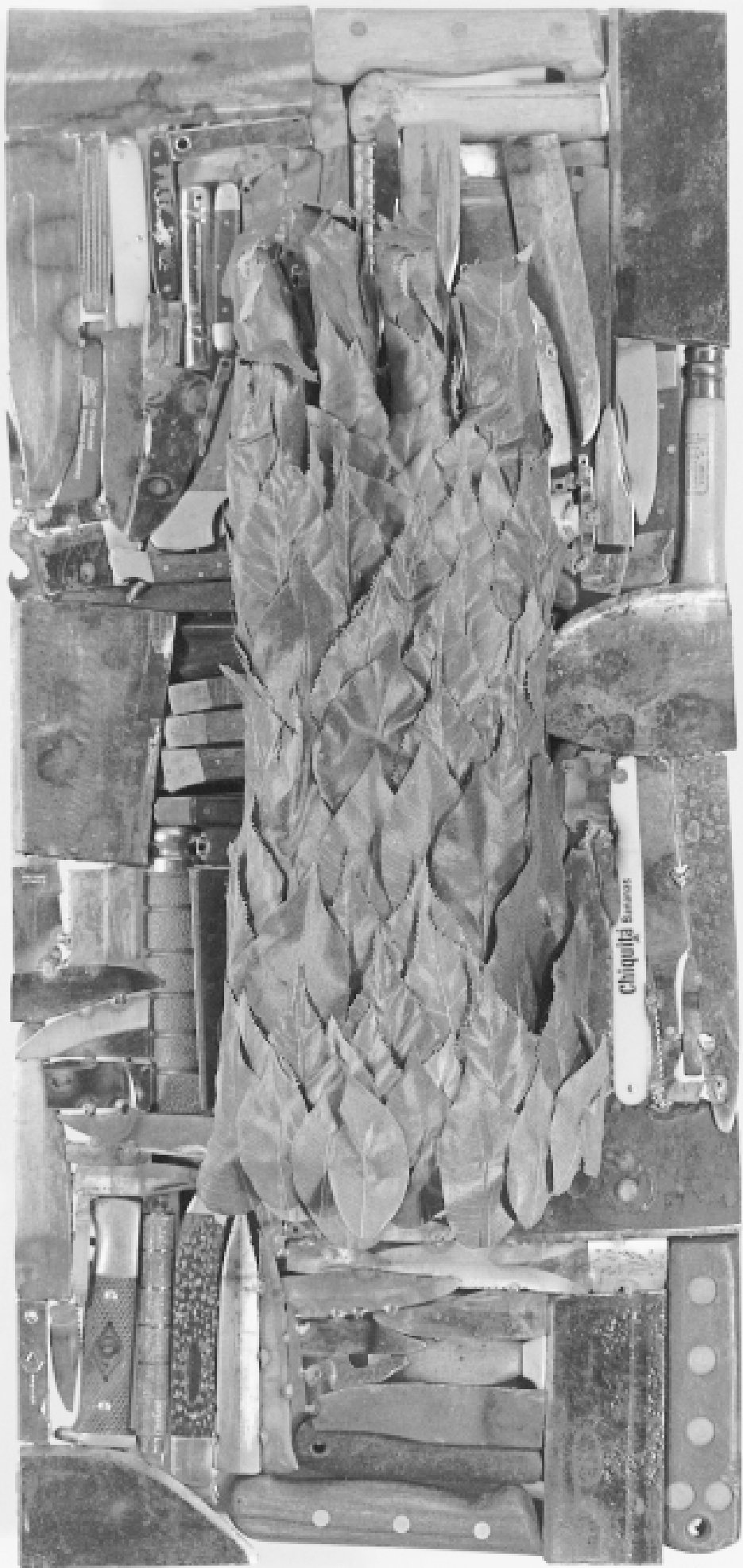

Alex Magrini, G ant de fer, 2001. Fragments d'armes blanches et feuilles synthétiques, 80 x 40 x $10 \mathrm{~cm}$. 


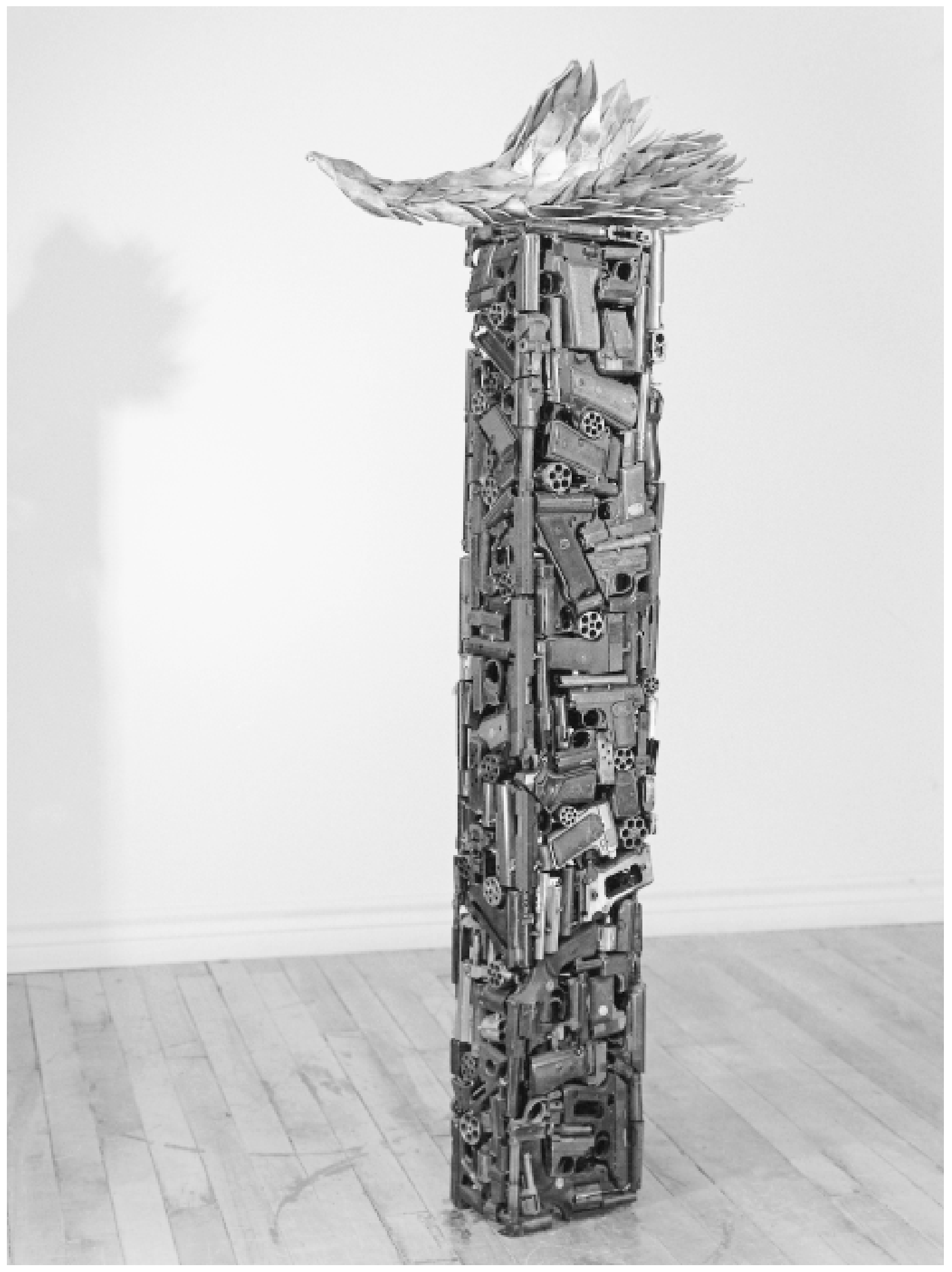

Alex Magrini, Apogée, 2000. Aluminium et fragments d'armes de poing, $30 \times 30 \times 140 \mathrm{~cm}$. 